\title{
THE TIME SCALE CALCULUS APPROACH TO THE GEODESIC PROBLEM IN 3D DYNAMIC DATA SETS
}

\author{
Sibel Paşalı Atmaca* and Ömer Akgüller \\ Department of Mathematics, Mugla Sitki Kocman University, 48000, Merkez, Mugla, \\ Turkey \\ *sibela@mu.edu.tr, oakguller@mu.edu.tr
}

\begin{abstract}
Geodesics have a fundamental role in the geometry of curved surfaces, as well as in discrete geometry. We present the time scale analogy of the dynamic data sets parameterized by a tensor product of two times scales. The goal of our study is the find the shortest and straightest path between two points on a point cloud like data sets which also involves continuous data.
\end{abstract}

Key Words- Discrete Differential Geometry, Time Scale Calculus, Discrete Geodesic Problem, Point Cloud Data

\section{INTRODUCTION}

Geodesics on smooth surfaces can generalize the idea of straight lines whose length does not decrease if it is perturbed in a small neighborhood of any point (disrupted in a small area at any point). From the differential equations viewpoint, geodesics solve the initial value problem which states that from any point of a manifold there starts a unique geodesic in any direction. On smooth surfaces geodesics possess both properties simultaneously, in contrast to the situation in discrete geodesic cases.

In discrete differential geometric concepts, where the polyhedral surfaces play a distinguished role, the geodesic problem can be considered in two manners; as "straightest" and "shortest" curves on triangular mesh elements.

Proposition 1.1 The concepts of straightest and shortest geodesics differ in the following way:

1) A geodesic $\gamma$ containing no surface vertex is both shortest and straightest.

2) A straightest geodesic through a spherical vertex is not locally shortest.

3) There exists a family of shortest geodesics through a hyperbolic vertex. Exactly one of them is a straightest geodesic.

The concept of shortest geodesics in polyhedral manifolds, and more general Sobolev spaces has been studied by researchers in many fields [1-6]. For applications related to vector fields, this concept has a central missing property, i.e. the initial value problem for geodesics has no unique solution and in hyperbolic vertex cases, has no solution. In [7] authors briefly describe the discrete geodesic problem as follows:

Theorem 1.2 (Discrete IVP) Let $M_{h}$ be a polyhedral surface and $p \in M_{h}$ a point with polyhedral tangent vector $v \in T_{p} M_{h}$. Then there exists a unique straightest geodesic $\gamma$ with

$\gamma(0)=p$

$\gamma^{\prime}(0)=v$, 
and the geodesic extends to the boundary of $M_{h}$.

In this study, we consider regularized point clouds as the discrete manifold in ambient space $R^{3}$, and define the geodesics on them. By using the unification idea of time scale calculus, we consider those geodesics as "shortest" with the variation of the length for a path on data set and "straightest" with the analogues of discrete IVP on time scales. Although we treat the case of data sets in $R^{3}$ in this paper, it is easy to generalize the idea to sub manifolds of $R^{N}$.

\section{METHOD}

The time scale calculus introduced by Stephan Hilger is a powerful tool to unify discrete and continuous analyses. Since it involves both characteristics of continuous and discrete ideas, it is also superior for discretization processes. For the sake of simplicity, we will refer you to such distinguish studies as [8-9] for the theory of time scale calculus. Also such studies as [10-12] are helpful to get into the geometry behind this theory, and one may take in account as the first geometric discretization step by using time scale calculus. The most significant difference for the theory arises from the product rule for delta derivative. This difference also leads us to the non-commutative geometry of the Quantum universe. Because of the lack of instrumental and computational possibilities, we avoid geometry with $q$-integers. This is the unique and attractive example of time scales throughout this study.

The term "dynamic data sets" comes from the idea of dynamic behavior of time.

Definition 2.1: Let $\mathrm{T}_{1}$ and $\mathrm{T}_{2}$ be two arbitrary time scales. The tensor product

$$
\Lambda^{2}=\mathrm{T}_{1} \times \mathrm{T}_{2}=\left\{(\mathrm{t}, \mathrm{s}) \mid \mathrm{t} \in \mathrm{T}_{1}, \mathrm{~s} \in \mathrm{T}_{2}\right\}
$$

is called dynamic data set in 2D. Moreover, let us define such map $\varphi: \Lambda^{2} \rightarrow R^{3}$ which satisfies the property of $\Delta$-regularity, i.e, $\frac{\partial \varphi}{\Delta_{1} \mathrm{t}} \times \frac{\partial \varphi}{\Delta_{2} \mathrm{~S}} \neq 0$. Therefore the set

$$
\Lambda^{3}=\{(\mathrm{t}, \mathrm{s}, \varphi(\mathrm{t}, \mathrm{s})\}
$$

is called dynamic data set in 3D.

It's easy to see that $\Lambda^{2}$ and $\Lambda^{3}$ are both closed subsets of $R^{2}$ and $R^{3}$, respectively. By the $\Delta$-regularity feature, it's possible to put such a vector space into the dynamic data set with respect to partial differences.

\subsection{The Geodesic Dynamic Equation}

Consider 3D dynamic data set $\Lambda^{3}$, with the parametric map $\varphi: \Lambda^{2} \rightarrow \Lambda^{3}$. We assume that $\varphi$ is a $\Delta$-smooth immersion, i.e., the delta differential of $\varphi$ is injective for all $x \in \Lambda^{2}$.

Definition 2.2 Let $\tilde{T}$ be a time scale. The function $\gamma$ is called a path on $\Lambda^{2}$ if it is increasing. Moreover, the composite function $\varphi \circ \gamma: \widetilde{T} \rightarrow \Lambda^{3}$ is called a path on 3D dynamic data set. 
The length of this path on $\Lambda^{3}$ can be computed by the means of metric tensor on the data set as follows:

$$
L_{\Lambda^{3}}(\gamma)=\int_{\mathrm{a}}^{\mathrm{b}}\left\|\frac{\mathrm{d}(\varphi \circ \gamma)}{\widetilde{\Delta} \mathrm{t}}\right\| \widetilde{\Delta} \mathrm{t}
$$

,where $\widetilde{\Delta}$ is the delta differential operator of $\tilde{T}$ and $t \in[a, b] \subset \widetilde{T}$. Since $\gamma$ is an increasing function, it's possible to apply the chain rule. Hence

$$
\begin{aligned}
L_{\Lambda^{3}}(\gamma) & =\int_{\mathrm{a}}^{\mathrm{b}}\left\|\bar{\Delta} \varphi_{\gamma(\mathrm{t})} \cdot \widetilde{\Delta} \gamma(\mathrm{t})\right\| \widetilde{\Delta} \mathrm{t} \\
& =\int_{\mathrm{a}}^{\mathrm{b}} \sqrt{\widetilde{\Delta} \gamma(\mathrm{t})^{\mathrm{T}} \cdot \bar{\Delta} \varphi_{\gamma(\mathrm{t})}^{\mathrm{T}} \bar{\Delta} \varphi_{\gamma(\mathrm{t})} \cdot \widetilde{\Delta} \gamma(\mathrm{t})} \widetilde{\Delta} \mathrm{t}
\end{aligned}
$$

,where $\bar{\Delta}$ is the delta differential operator of $\bar{T}=\gamma(\tilde{T})$.

This result leads us to obtain an inner product at $u=\varphi(p) \in \Lambda^{3}$ defined by

$$
\begin{aligned}
g_{u}(v, w) & =\left\langle\bar{\Delta} \varphi_{\mathrm{u}} \mathrm{v}, \bar{\Delta} \varphi_{\mathrm{u}} \mathrm{w}\right\rangle \\
& =v^{t} \bar{\Delta} \varphi_{\mathrm{u}}^{\mathrm{T}} \bar{\Delta} \varphi_{\mathrm{u}} \mathrm{w}
\end{aligned}
$$

Using this inner product involves a tensor metric; it's possible to write the length of a path on data set as

$$
L_{\Lambda^{3}}(\gamma)=\int_{a}^{b} \sqrt{g_{\gamma(t)}\left(\gamma^{\widetilde{\Delta}}(t), \gamma^{\widetilde{\Delta}}(t)\right)} \widetilde{\Delta} \mathrm{t}
$$

intrinsically. For $\gamma(t) \in \Lambda^{2}$, we let $G_{\gamma(t)}$ be the 2 by 2 matrix $\widetilde{\Delta} \gamma(\mathrm{t})^{\mathrm{T}} \widetilde{\Delta} \gamma(\mathrm{t})$ corresponding to the inner product $g_{\gamma(t)}$. Observe that, $G_{\gamma(t)}$ is invertible since it corresponds to a positive definite (and hence non-degenerate) inner product.

To obtain the shortest characterization of geodesic paths on $\Lambda^{3}$, we first need to analyze the variation of the paths between given points and then construct the system of dynamic equations.

Theorem 2.3: Let $\Gamma=\varphi \circ \gamma$ be a $\Delta$-differentiable path and $\Gamma_{\lambda}: \widetilde{T} \rightarrow \Lambda^{3}$ be the family of paths on $\Lambda^{3}$ with $|\lambda|<\varepsilon, \Gamma_{0}=\Gamma$. Then we have

\section{Proof}

$$
\frac{d}{d \lambda} L_{\Lambda^{3}}(\Gamma)=-\int_{\mathrm{a}}^{\mathrm{b}}\left(\left.\frac{d}{d \lambda}\right|_{0} \Gamma_{\lambda}(\tilde{\sigma}(s))\right) \Gamma^{\widetilde{\Delta} \widetilde{\Delta}}(\mathrm{s}) \widetilde{\Delta} \mathrm{s} .
$$

$$
\frac{d}{d \lambda} L_{\Lambda^{3}}(\Gamma)=\int_{\mathrm{a}}^{\mathrm{b}}\left\|\Gamma_{\lambda}^{\widetilde{\Delta}}(\mathrm{s})\right\| \widetilde{\Delta} \mathrm{s}=\int_{\mathrm{a}}^{\mathrm{b}} \frac{\mathrm{d}}{\mathrm{d} \lambda} \sqrt{\Gamma_{\lambda}^{\widetilde{\Delta}}(\mathrm{s}) \Gamma_{\lambda}^{\widetilde{\Delta}}(\mathrm{s})} \widetilde{\Delta} \mathrm{s}
$$




$$
\begin{aligned}
& =\int_{a}^{b}\left(\frac{d}{d \lambda} \Gamma_{\lambda}^{\widetilde{\Delta}}(\mathrm{s})\right) \Gamma_{\lambda}^{\widetilde{\Delta}}(\mathrm{s}) \widetilde{\Delta} \mathrm{s} \\
& =-\int_{\mathrm{a}}^{\mathrm{b}} \Gamma^{\widetilde{\Delta} \widetilde{\Delta}}(\mathrm{s})\left(\left.\frac{d}{d \lambda}\right|_{0} \Gamma_{\lambda}(\tilde{\sigma}(s))\right) \widetilde{\Delta} \mathrm{s} .
\end{aligned}
$$

Theorem 2.5 A $\widetilde{\Delta}$-differentiable path $\Gamma$ parameterized by arc length is a geodesic path if and only if $\Gamma^{\widetilde{\Delta} \widetilde{\Delta}}(\mathrm{s})=0$ for all $s \in \widetilde{T}$. Moreover, let $\Lambda^{3}$ be a $3 \mathrm{D}$ dynamic data set. For all $p \in \Lambda^{3}$, and any $\Delta$-tangent direction $v$ the initial value problem

$$
\begin{aligned}
\Gamma^{\widetilde{\Delta} \widetilde{\Delta}}(\mathrm{s}) & =0 \\
\Gamma(0) & =p \\
\Gamma^{\widetilde{\Delta}}(0) & =\mathrm{v}
\end{aligned}
$$

has unique solution $\Gamma$.

Corollary 2.6: Let $\varphi: \Lambda^{2} \rightarrow \Lambda^{3}$ defined by $\varphi(u(t), v(t))$, where $t \in \tilde{T}, u \in T_{1}, v \in T_{2}$. Then $\varphi(u(t), v(t))$ is the shortest path between two points in $\Lambda^{3}$ if and only if following nonlinear system of partial dynamical equations is satisfied:

$$
\begin{aligned}
& 0=u^{\widetilde{\Delta} \widetilde{\Delta}}\left(\varphi^{\Delta_{1}}\right)^{2}+u^{\widetilde{\Delta} \widetilde{\sigma}} u^{\widetilde{\Delta}} \varphi^{\Delta_{1}} \varphi^{\Delta_{11}}+u^{\widetilde{\Delta} \widetilde{\sigma}} v^{\widetilde{\Delta}} \varphi^{\Delta_{1}} \varphi^{\Delta_{12}}+v^{\widetilde{\Delta} \widetilde{\sigma}} u^{\widetilde{\Delta}} \varphi^{\Delta_{1}} \varphi^{\Delta_{21}} \\
& +v^{\widetilde{\Delta} \widetilde{\sigma}} v^{\widetilde{\Delta}} \varphi^{\Delta_{1}} \varphi^{\Delta_{22}} \\
& 0=v^{\widetilde{\Delta} \widetilde{\Delta}}\left(\varphi^{\Delta_{2}}\right)^{2}+u^{\widetilde{\Delta} \widetilde{\sigma}} u^{\widetilde{\Delta}} \varphi^{\Delta_{2}} \varphi^{\Delta_{11}}+u^{\widetilde{\Delta} \widetilde{\sigma}} v^{\widetilde{\Delta}} \varphi^{\Delta_{2}} \varphi^{\Delta_{12}}+v^{\widetilde{\Delta} \widetilde{\sigma}} u^{\widetilde{\Delta}} \varphi^{\Delta_{2}} \varphi^{\Delta_{21}} \\
& +v^{\widetilde{\Delta} \widetilde{\sigma}} v^{\widetilde{\Delta}} \varphi^{\Delta_{2}} \varphi^{\Delta_{22}}
\end{aligned}
$$

\section{EXAMPLES}

For the numerical interpretation of our method, we solved the IVP given in Corollary 2.6 as solving a dynamic equation. Since there isn't any unified numerical method for dynamic equations, we considered our equation as difference and differential equation for the convenient data points, and then applied a finite difference method for the solutions. Geometric results are given in the following examples for different data sets.

Example 3.1. Let $T_{1}=\left\{t^{2} \mid t \in Z_{100}\right\}$ and $T_{2}=\left\{\sqrt{s} \mid s \in Z_{100}\right\}$. For $\varphi: \Lambda^{2} \rightarrow R^{3}$, let us define $\varphi(t, s)=\left(t \sqrt{s}, s+t^{3}, s-t\right)$ and $\varphi\left(\Lambda^{2}\right)=\Lambda^{3}$. Therefore it is possible to obtain the shortest path between the two arbitrary points we choose as follows: 


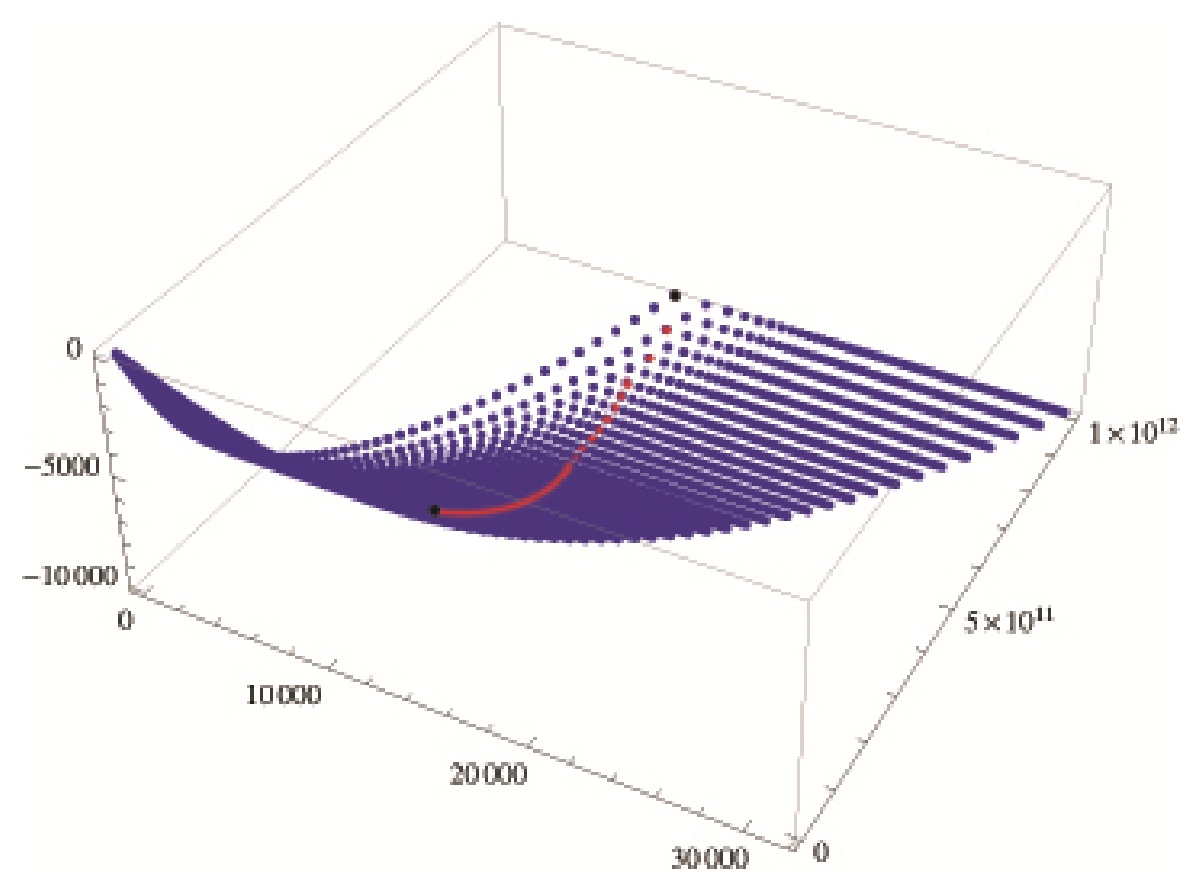

Example 3.2. Let $T_{1}=\left\{t \mid t \in Z_{100}\right\}$ and $T_{2}=\left\{s \mid s \in Z_{100}\right\}$. For $\varphi: \Lambda^{2} \rightarrow R^{3}$, let us define $\varphi(t, s)=\left(e_{1}(0, s), e_{1}(0, t), s / t\right)$, where $e_{p}\left(u_{0}, u\right)$ are exponential functions and $\varphi\left(\Lambda^{2}\right)=\Lambda^{3}$. To obtain the shortest path between the two arbitrary points we choose as follows in different directions:

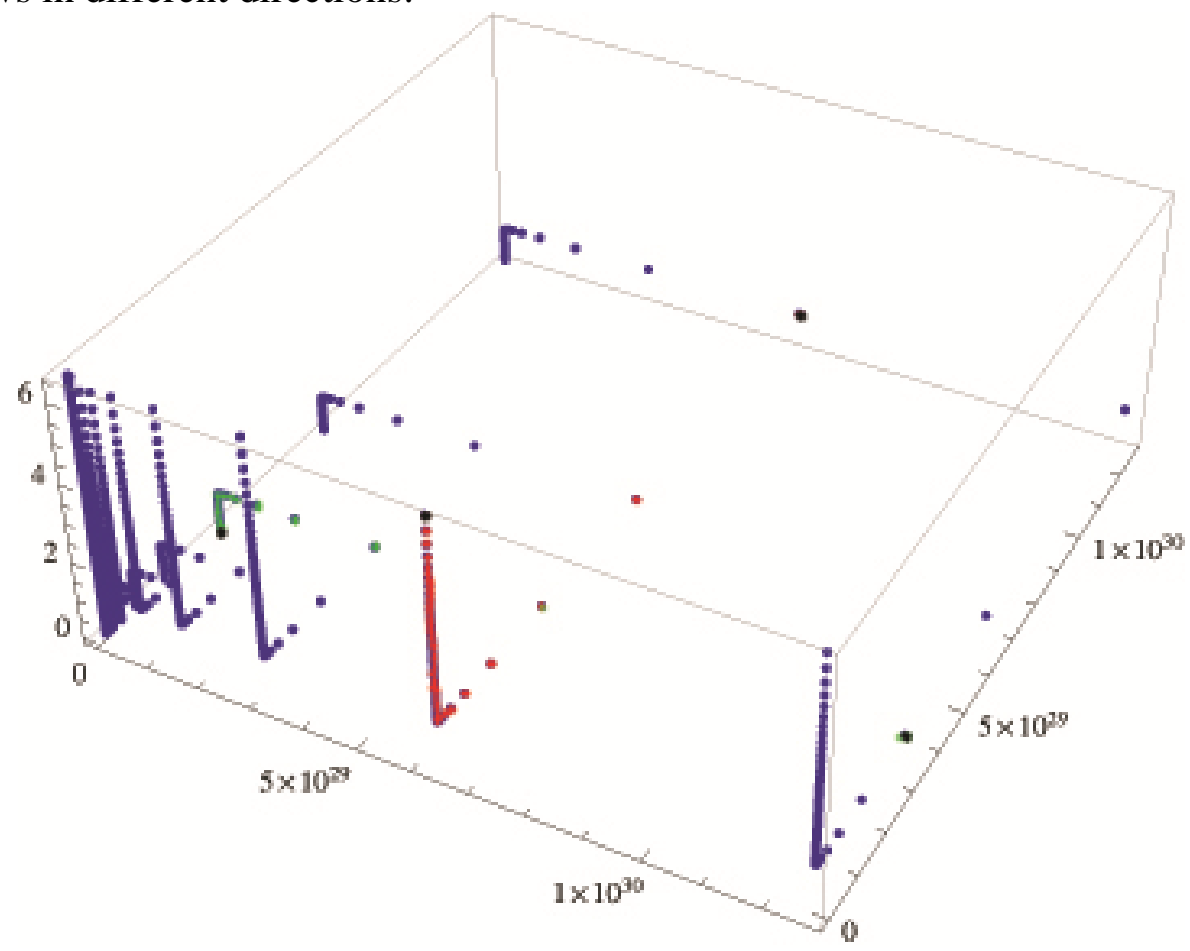

Example 3.2. Let $T_{1}=\left\{1 / t \mid t \in Z_{100}\right\}$ and $T_{2}=\left\{2 s \mid s \in Z_{100}\right\}$. For $\varphi: \Lambda^{2} \rightarrow R^{3}$, let us define $\varphi(t, s)=\left(s-t^{3}, t s, s^{2}-t\right)$, and $\varphi\left(\Lambda^{2}\right)=\Lambda^{3}$. To obtain the shortest path between the two arbitrary points we choose as follows in different directions: 


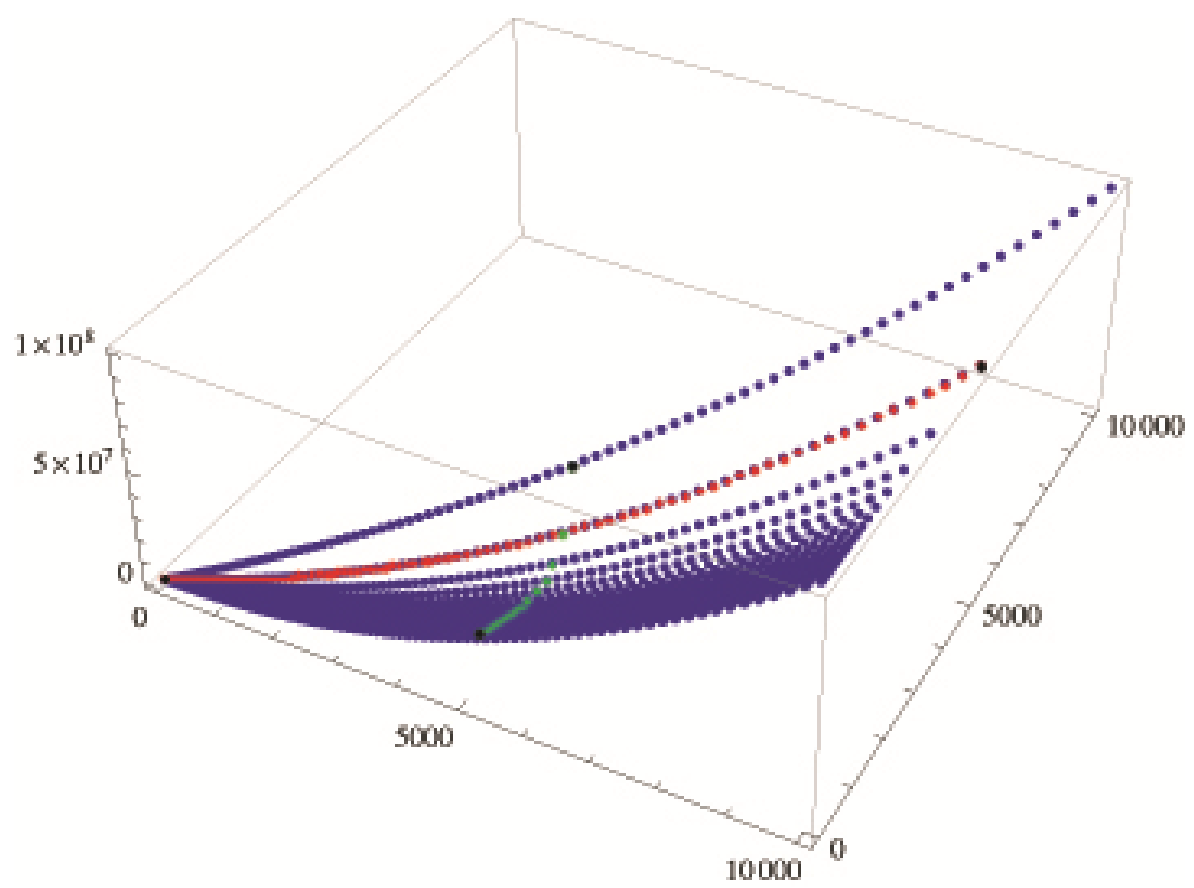

\section{DISCUSSION}

The method we present in this study is quiet new and may be considered as the first unification attempts of discrete and continuous geodesic problems. Although the dynamic data set we present is pretty regular, it's is possible to regularize the data sets of real world problems by well known methods and then apply the time scale approach to obtain the geodesic like curves and paths between two points before computer aided geometric design processes such as triangulation and rendering. Also the method is convenient to construct such linear splines between data points and apply well known graph path algorithms. With the help of linear splines one can also complete the region of discrete parts with the rectangular mesh elements and study other geometric properties such as flows, vector fields and bending energies. Topological changes and computational complexity are the most significant handicaps of our method. The fast graph algorithms on discrete parts and $\alpha$-shapes approach will be considered to solve those problems for future studies.

Acknowledgment-This research was supported and funded by The Scientific and Technological Research Council of Turkey (TUBITAK) (113T015).

\section{REFERENCES}

1. K. Hildebrandt, K. Polthier, M. Wardetzky, On the convergence of metric and geometric properties of polyhedral surfaces, Geom Dedicata 123, 89-112, 2006 
2. R. Kimmel, A. Amir, A.M. Bruckstein, Finding shortest paths on surfaces. In: P.J. Laurent, A. LeMéhauté, L.L. Schumaker, (Eds.), Curves and Surfaces in Geometric Design. A.K. Peters, Wellesley, MA, 259-268, 1994

3. R. Kimmel, J. Sethian, Computing geodesic paths on manifolds, Proc. Natl. Acad. Sci. 95(15), 8431-8435, 1995.

4. T. Maekawa, Computation of shortest paths on free-form parametric surfaces, Journal of Mechanical Design 118, 499-508, 1996.

5. F. Memoli, G. Sapiro, Distance functions and geodesics on submanifolds of Rd and point clouds, SIAM Journal on Applied Mathematics 65(4), 1227-1260, 2005.

6. Jr. D.D. Streeter, Gross morphology and fiber geometry of the heart. In: Berne, R.M., Sperelakis, N. (Eds.), Handbook of Physiology, The Heart. American

Physiology Society, Williams and Wilkins, Baltimore, pp. 61-112, 1979.

7. M. Schmies, K. Polthier, Straightest geodesics on polyhedral surfaces, Mathematical Visualization, 1998.

8. M. Bohner and G. Sh. Guseinov, Partial differentiation on time scales, Dynamic Systems and Applications 13(3-4), pp. 351379, 2004.

9. M. Bohner and A. Peterson, Dynamic Equations on Time Scales: An Introduction with Applications, Birkhauser, Boston, Mass, USA, 2001.

10. G. Sh. Guseinov and E. Ozyilmaz, Tangent lines of generalized regular curves parametrized by time scales, Turkish Journal of Mathematics 25(4) 553562, 2001.

11. E. Ozyilmaz, Directional Derivative of Vector Field and Regular Curves on Time Scales, Applied Mathematics and Mechanics (English Edition) 27(10) 1349-1360, 2006. 12. S. P. Atmaca, Normal and Osculating Planes of Regular Curves, Abstract and Applied Analysis 2010, doi:10.1155/2010/923916, 2010. 